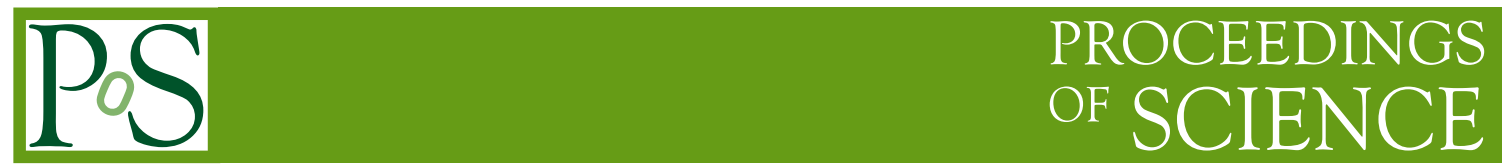

\title{
$\left|V_{u d}\right|,\left|V_{u s}\right|,\left|V_{c d}\right|,\left|V_{c s}\right|$ and charm (semi)leptonic decays: WG1 summary from CKM 2016
}

\section{Takashi Kaneko}

High Energy Accelerator Research Organization (KEK), Ibaraki 305-0801, Japan

School of High Energy Accelerator Science, SOKENDAI (The Graduate University for Advanced Studies), Ibaraki 305-0801, Japan

E-mail: takashi.kaneko@kek.jp

\section{Xiao-Rui Lyu}

University of Chinese Academy of Sciences, Beijing 100049, China

E-mail: xiaorui@ucas.ac.cn

\section{Arantza Oyanguren}

Instituto de Fisica Corpuscular, Centro Mixto Universidad de Valencia - CSIC, Valencia, Spain

E-mail: Arantza. Oyangureneific.uv.es

We summarize the current status of the determination of the CKM matrix elements $\left|V_{u d}\right|$ and $\left|V_{u s}\right|$, which is at the precision frontier of CKM phenomenology. We also review recent progress on the study of charm (semi)leptonic decays, and the determination of $\left|V_{c d}\right|$ and $\left|V_{c s}\right|$.

9th International Workshop on the CKM Unitarity Triangle

28 November - 3 December 2016

Tata Institute for Fundamental Research (TIFR), Mumbai, India 


\section{Introduction}

The precise determination of the Cabibbo-Kobayashi-Maskawa (CKM) matrix elements is one of the most important subjects in the search for new physics. New bounds on violations of CKM unitarity, for instance, translate into constraints on models beyond the Standard Model (SM), and may eventually turn up evidence of new physics. Due to the CKM hierarchy, the elements $\left|V_{u d}\right|$, $\left|V_{u s}\right|,\left|V_{c d}\right|$ and $\left|V_{c s}\right|$ have (sub-)dominant contributions to the unitarity condition in the first and second rows.

At present, the first row condition $\left|V_{u d}\right|^{2}+\left|V_{u s}\right|^{2}+\left|V_{u b}\right|^{2}=1$ provides the most stringent test of CKM unitarity. The relevant elements, $\left|V_{u d}\right|$ and $\left|V_{u s}\right|$, have been precisely determined from the super-allowed nuclear $\beta$ decays and $K \rightarrow \pi \ell v$ decays, respectively. An advantage in controlling hadronic uncertainties lies in the fact that these decays proceed through the weak vector current. The conservation of the vector current (CVC) and the non-renormalization theorem imply that the relevant matrix elements at zero momentum transfer are known in the isospin (SU(3)) limit. Corrections to the symmetric limit are quadratic in a symmetry-breaking parameter $m_{d(s)}-m_{u}[1,2]$.

It is of course important to explore various different decays. Such decays provide not only independent determinations of the CKM elements but also complementary probes of new physics. For instance, the weak axial current contributes to the neutron $\beta$ decays, kaon and pion leptonic decays, which are therefore sensitive to pseudoscalar- and axial-vector-type new physics interactions. Inclusive hadronic $\tau$ decays may be sensitive to new physics that couples primarily to the third generation.

There are a rich variety of charm decay modes, which are important in the search for new physics. The $D_{(s)} \rightarrow \ell v$ and $D \rightarrow \pi(K) \ell v$ decays provide a precise determination of $\left|V_{c d(s)}\right|$. Rare and forbidden decay modes may serve as sensitive probes of new physics. Large data samples of the charm decays are being accumulated at charm and $B$ factories leading to a recent remarkable improvement in the experimental accuracy. Control of the hadronic uncertainties is more difficult than that in the kaon decays due to the large flavor symmetry breaking. Thanks to the continuous development of powerful computers and simulation algorithms, the accuracy of lattice QCD determinations of the hadronic matrix elements has also been improved in recent years.

In this summary, we present an overview of recent progress reported in the Working Group 1 sessions of the CKM 2016 workshop.

\section{2. $\left|V_{u d},\right| V_{u s} \mid$ and unitarity in the first row}

\section{1 $\left|V_{u d}\right|$ from nuclear and neutron decays}

The super-allowed nuclear $\beta$ decays, namely $0^{+} \rightarrow 0^{+}$transitions of isospin-one $(I=1)$ nuclei, have provided the most precise determination of $\left|V_{u d}\right|$. A key quantity is the product of the phase space factor $f$ and the partial half-life $t$. By eliminating transition-dependent corrections $\delta_{R}^{\prime}, \delta_{N S}$ and $\delta_{C}$, we can define a corrected value

$$
\mathscr{F} t=f t\left(1+\delta_{R}^{\prime}\right)\left(1+\delta_{N S}-\delta_{C}\right)=\frac{K}{2 G_{V}^{2}\left(1+\Delta_{R}\right)},
$$



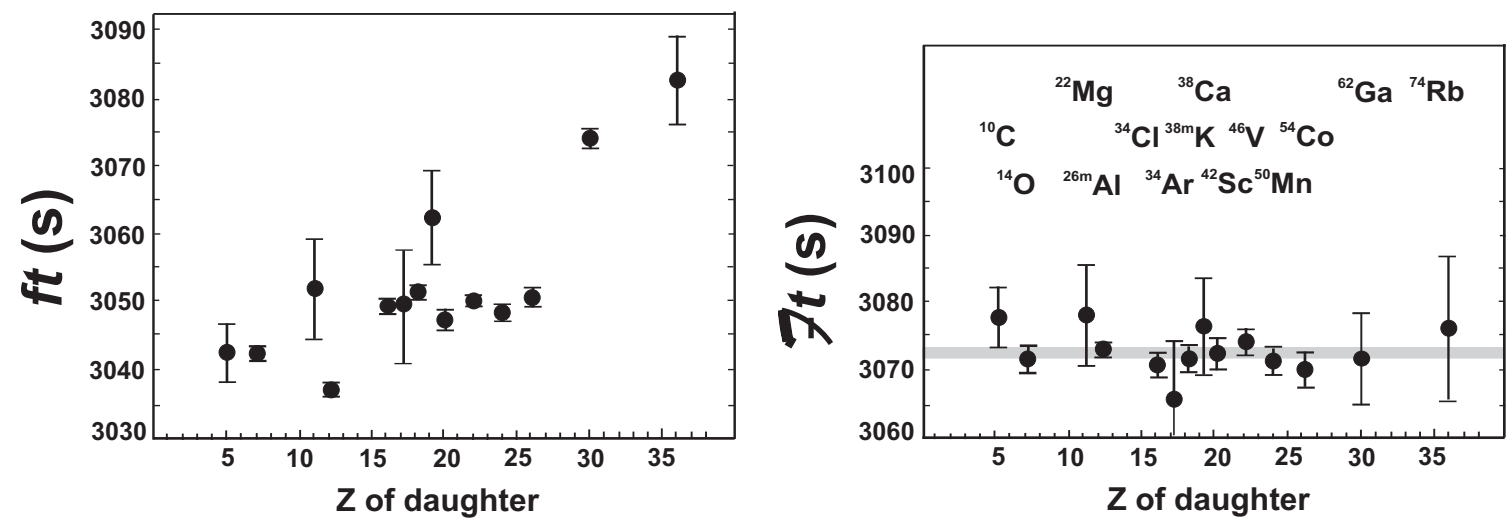

Figure 1: Results for the uncorrected $f t$ values (left panel) and corrected $\mathscr{F} t$ values (right panel) for the 14 best-known super-allowed nuclear $\beta$ decays. (Figure from Ref. [3].)

which is expected to be transition independent. Here $\delta_{R}^{\prime}$ and $\delta_{N S}$ represent the radiative corrections, whereas $\delta_{C}$ is the isospin-symmetry breaking correction. Note that $\delta_{N S}$ and $\delta_{C}$ depend on the details of nuclear structure. In the right-hand side, $\Delta_{R}$ represents the transition-independent radiative correction. The vector coupling $G_{V}$ is related to $\left|V_{u d}\right|$ and the Fermi coupling $G_{F}$ as $G_{V}=\left|V_{u d}\right| G_{F}$. Since the constant $K$ and $G_{F}$ have negligibly small uncertainties, we can obtain $\left|V_{u d}\right|$ from experimental determination of $f t$ and theoretical calculation of $\delta_{C}, \delta_{N S}, \delta_{R}$ and $\Delta_{R}$.

As reviewed by J.C. Hardy [3], new experimental results became available after the last workshop CKM 2014: the total transition energy and branching ratio for the decay branch from ${ }^{14} \mathrm{O}$, and the half-life of ${ }^{10} C[4,5,6]$, which are inputs to determine $f t$. The left panel of Fig. 1 shows the updated average of $f t$ for the 14 best-known decays [3]. The precision is $\leq 0.05 \%$ for the nine $f t$ values, and $<0.3 \%$ for the other five cases.

The horizontal axis of the panel represents the atomic number $Z$ of the daughter nucleus. The $Z$ dependence of $f t$ is small (note the vertical axis scale of the figure) but significant at the high precision of $f t$. Among the transition-dependent corrections, the isospin correction $\delta_{C}$ becomes importantly large as $Z$ increases. Several methods have been proposed to calculate $\delta_{C}$ [7]. Only the shell-model calculation using the so-called Saxon-Woods radial wave-function leads to the impressive agreement of the corrected $\mathscr{F} t$ values as shown in the right panel of Fig. 1. This confirms the CVC expectation of a universal value of $G_{V}$ at the level of $\pm 0.01 \%$.

The accuracy of $\mathscr{F} t$ is then further improved by averaging over $Z$. Theoretical corrections are small (partly) due to CVC. These decays therefore yields very precise estimate:

$$
\left|V_{u d}\right|=0.97420(21) \quad \text { (super-allowed nuclear decays). }
$$

This $0.02 \%$ uncertainty is dominated by that from the calculation of the transition independent correction $\Delta_{R}$. Therefore, only little reduction of this uncertainty is possible without improved calculation of $\Delta_{R}$.

The neutron $\beta$ decays receive the radiative corrections $\delta_{R}^{\prime}$ and $\Delta_{R}$, but they are free of the nucleus-dependent corrections $\delta_{N S}$ and $\delta_{C}$, which depend on the nucleus structure. These decays 

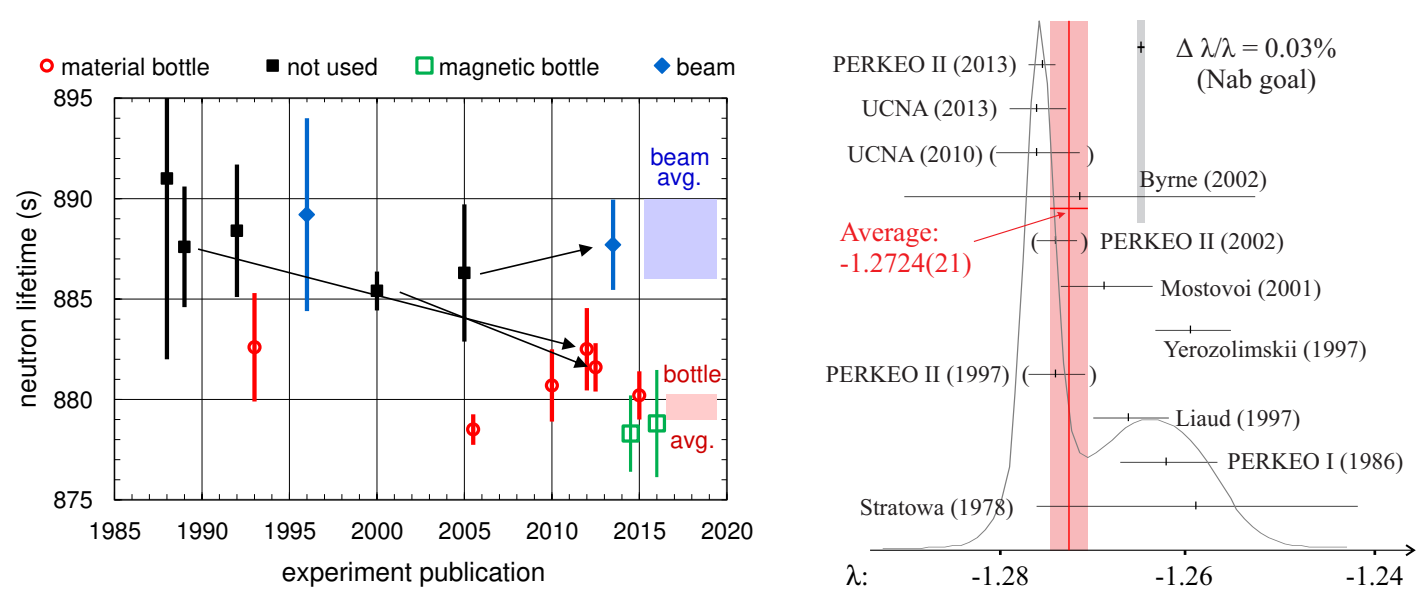

Figure 2: Left panel: neutron lifetime measurements using the beam method (diamonds) and bottle method (circles and squares). Two shaded regions show the averages of $\tau_{n}$ from two methods. Right panel: measurements of $\lambda$. The red band shows its global average, whereas the planned precision of the upcoming Nab experiment [12] is shown in the gray band. (Figure from Ref. [8].)

may therefore be better to determine $\left|V_{u d}\right|$ in the long term. The master formula is

$$
\left|V_{u d}\right|^{2}=\frac{4908.7(1.9)[s]}{\tau_{n}\left(1+3 \lambda^{2}\right)}
$$

where $\tau_{n}$ is the neutron lifetime. In contrast to the super-allowed nuclear decays, the neutron decays proceed also through the weak axial current. These decays therefore provide an independent determination of $\left|V_{u d}\right|$ with different sensitivity to new physics. However, the vector and axialvector contributions have to be disentangled through a difficult decay correlation measurement to fix $\lambda=G_{A} / G_{V}$, where $G_{A}$ is the axial coupling. A target accuracy is $0.02-0.03 \%$ both for $\tau_{n}$ and $\lambda$ to be competitive to the determination from the super-allowed nuclear decays. The current status and future prospect are summarized by D. Počanić in Ref. [8].

The neutron lifetime $\tau_{n}$ has been measured by two methods [9]. In the beam method [10], the number of neutron decays is counted as a cold neutron beam passes through a fiducial volume. On the other hand, the bottle method [11] stores ultra-cold neutrons in a material or magnetogravitational bottle, and counts the survivors after some storage time. As shown in the left panel of Fig. 2, however, averages of $\tau_{n}$ from these two methods appear to be systematically different from each other: 888.0(2.0) $s$ and 879.6(0.6) $s$ from the beam and bottle methods, respectively. Underestimated systematic uncertainties or unknown effects are the most likely cause of this $4 \sigma$ discrepancy, which is to be understood by forthcoming experiments [8].

The ratio $\lambda$ can be independently extracted from three correlation coefficients in the neutron decay rate [13]: the $\beta$ asymmetry $A, v_{e}$ asymmetry $B$, and $e-v_{e}$ correlation coefficient $a$. It is known that $A$ and $a$ have comparable sensitivities to $\lambda$, while $B$ is much less sensitive. So far, $A$ has been measured more precisely than $a$, and yields the most accurate results for $\lambda$. However, the world data of $\lambda$ in the right panel of Fig. 2 are not perfectly consistent among themselves and lead to the global average $\lambda=-1.2724(21)$ with a poor confidence level.

These inputs yields $\left|V_{u d}\right|=0.9758$ (16) with significantly larger uncertainty compared to Eq.(2.2). As listed in Ref. [8], however, there are many on-going and planned measurements both for $\tau_{n}$ and 
$\lambda$ to resolve the discrepancies among different methods and to improve the accuracy of $\left|V_{u d}\right|$.

\section{$2.2\left|V_{u s}\right|$ from kaon and $\tau$ decays}

The $K \rightarrow \pi \ell v$ semileptonic decays, namely the $K_{l 3}$ decays, provide a precise determination of $\left|V_{u s}\right|$. The decay rate is given by

$$
\Gamma_{K_{\ell 3}}=\frac{G_{F}^{2} M_{K}^{5}}{192 \pi^{3}} C_{K}^{2} I_{K \ell} S_{\mathrm{EW}}\left(1+\delta_{\mathrm{EM}}^{K \ell}+\delta_{\mathrm{SU}(2)}^{K \pi}\right)^{2}\left|V_{u s} f_{+}^{K^{0} \pi^{-}}(0)\right|^{2},
$$

where $C_{K}$ is a Clebsch-Gordan coefficient and $I_{K \ell}$ is the phase-space integral. The short-distance electroweak correction is given by $S_{\mathrm{EW}}=1.0232(3)$. The long-distance electromagnetic (EM) and isospin corrections are denoted by $\delta_{\mathrm{EM}}^{K \ell}$ and $\delta_{\mathrm{SU}(2)}^{K \pi}$, respectively, and have been estimated based on chiral perturbation theory (ChPT). The relevant form factors are defined from the hadronic matrix element

$$
\left\langle\pi\left(p^{\prime}\right)\left|V_{\mu}\right| K(p)\right\rangle=\left\{p+p^{\prime}-\frac{M_{K}^{2}-M_{\pi}^{2}}{q^{2}} q\right\}_{\mu} f_{+}^{K \pi}\left(q^{2}\right)+\frac{M_{K}^{2}-M_{\pi}^{2}}{q^{2}} q_{\mu} f_{0}^{K \pi}\left(q^{2}\right),
$$

where $q^{2}=\left(p^{\prime}-p\right)^{2}$ is the momentum transfer. Only the vector component of the weak current contributes to these decays, and SU(3) breaking effects to $f_{+}^{K \pi}(0)$ is second order in $m_{s}-m_{u}$ [2].

The kaon leptonic decays $\left(K_{\ell 2}\right)$, on the other hand, proceed through the weak axial current. The relevant hadronic input, the decay constant $f_{K}$, breaks SU(3) invariance already at first order. This and other corrections can partially cancel in the decay rate ratio to the pion decays $\left(\pi_{\ell 2}\right)$

$$
\frac{\Gamma_{K_{\ell 2}}}{\Gamma_{\pi_{\ell 2}}}=\frac{M_{K}\left(1-m_{\ell}^{2} / M_{K}^{2}\right)^{2}}{M_{\pi}\left(1-m_{\ell}^{2} / M_{\pi}^{2}\right)^{2}}\left(1+\delta_{\mathrm{EM}}\right) \frac{\left|V_{u s}\right|^{2}}{\left|V_{u d}\right|^{2}} \frac{f_{K}^{2}}{f_{\pi}^{2}},
$$

where $\delta_{\mathrm{EM}}$ is the long-distance $\mathrm{EM}$ correction. These $P_{\ell 2}(P=K, \pi)$ decays therefore provide an independent determination of $\left|V_{u s}\right| /\left|V_{u d}\right|$ [14].

The experimental inputs, $\Gamma_{\left\{K_{\ell 3}, K_{\ell 2}\right\}}$ and $I_{K \ell}$, were precisely measured by kaon experiments between 2003 and 2010 [15]. After the last workshop, there are no significant new experimental inputs with full error budget. Only the ChPT estimate of $\delta_{\mathrm{SU}(2)}^{K \pi}$ has been slightly changed with updated inputs (quark mass ratios) from lattice QCD [16]. The values $\left|V_{u s} f_{+}(0)\right|=0.21654(41)$ and $\left|V_{u s}\right| f_{K} /\left|V_{u d}\right| f_{\pi}=0.27599$ (37) remain essentially unchanged [17]. As reviewed by M. Moulson in detail, there are good prospects for a wealth of new experiments. For instance, the $0.19 \%$ uncertainty of $\left|V_{u s} f_{+}(0)\right|$ may be reduced to $\approx 0.12 \%$ within the next five years [17].

As emphasized by S. Simula [18], the accuracy of the lattice QCD determination of the hadronic inputs has been steadily improved by large-scale simulations near (or even at) the physical quark masses on fine and large lattices. The left panel of Fig. 3 presents a compilation of such realistic simulations. The current world averages for $N_{f}=2+1+1$ QCD,$f_{K} / f_{\pi}=1.1933$ (29) [16] and $f_{+}(0)=0.9706(27)$ [19], have now the total uncertainty of $0.3 \%$, and yield

$$
\left|V_{u s}\right|=0.2231(9), \quad \frac{\left|V_{u s}\right|}{\left|V_{u d}\right|}=0.2313(6) \quad\left(K_{\ell 3} \text { and } P_{\ell 2} \text { decays }\right) .
$$

At the impressive accuracy of the hadronic inputs, the uncertainty of the EM and isospin corrections, which is typically $0.1-0.4 \%$ [15], is no longer negligible. It is difficult to extend the 

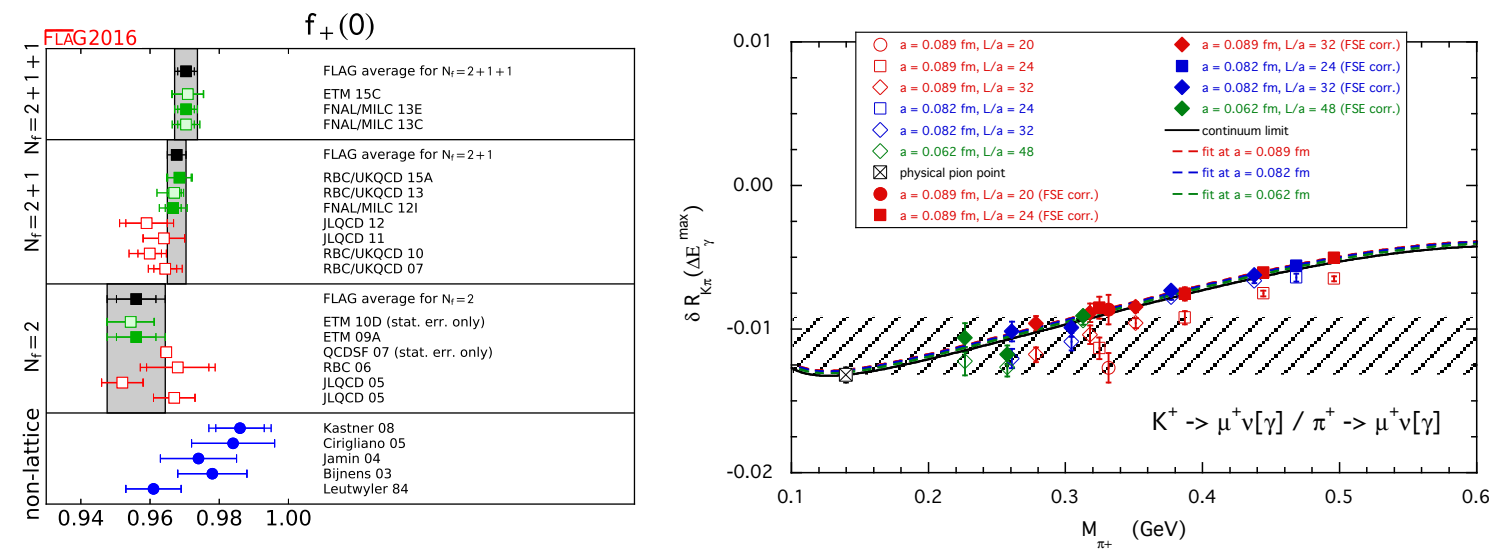

Figure 3: Left panel: lattice determination of $f_{+}(0)$ in $N_{f}=2,2+1$ and 2+1+1 QCD (squares) and phenomenological estimate (circles). The black square shows the average for each $N_{f}$. (Figure from Ref. [16].) Right panel: EM and isospin correction to the decay rate ratio (2.6). Lattice simulation results are plotted by symbols, and lines are their fit curves at finite lattice spacings and in the continuum limit. The shaded band represents the ChPT estimate [22, 23]. (Figure from Ref. [21].)

ChPT calculation to higher orders where many additional unknown low energy constants appear. Recently, a new strategy was proposed to calculate the EM correction on the lattice for hadronic processes, where infrared divergence are present [20]. This has been succesfully applied to the $P_{\ell 2}$ decays [21]: their preliminary estimate of $\delta_{\mathrm{EM}}$ plus the isospin correction to $f_{K} / f_{\pi}$ is $\delta R_{K \pi}=$ $-0.0137(13)$, which is in good agreement with the ChPT estimate $-0.0112(21)[22,23]$ as shown in the right panel of Fig. 3.

The inclusive hadronic $\tau$ decays offer an alternative determination of $\left|V_{u s}\right|$ [24]. Previous estimate at the time of the CKM 2014 workshop is $\left|V_{u s}\right|=0.2176(21)$ [25], and about $3 \sigma$ below the value quoted in Eq. (2.7). This determination employs the finite energy sum rule to estimate the partial inclusive decay rate $\Gamma\left(\tau \rightarrow X_{\{s, d\}} v_{\tau}\right)$. A key relation is

$$
\begin{aligned}
\int_{0}^{s_{0}} d s & w(s) \Delta \rho_{\tau}(s) \\
& =-\frac{1}{2 \pi i} \oint_{|s|=s_{0}} d s w(s) \Delta \Pi_{\tau}(s),
\end{aligned}
$$

where $s$ denotes the momentum transfer to the hadronic state $X_{\{s, d\}}$. The spectral function $\Delta \rho_{\tau}$ is experimentally accessible from the differential

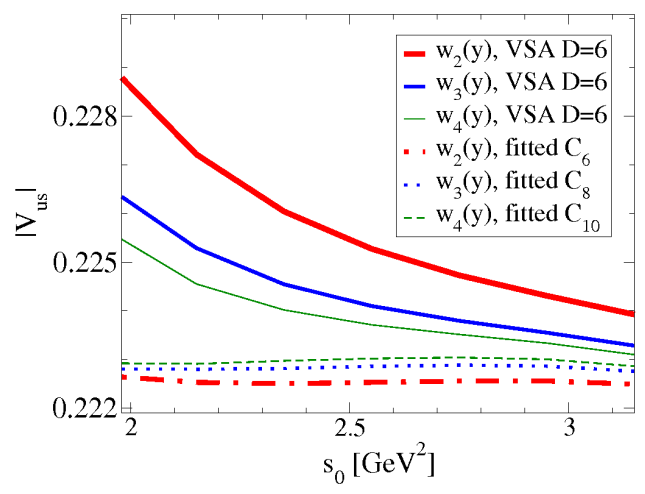

Figure 4: Estimate of $\left|V_{u s}\right|$ as a function of $s_{0}$. Three different weight functions $w_{\{2,3,4\}}$ are tested in the conventional (solid lines) and new implementations (dot-dashed, dotted and dashed lines). (Figure from Ref. [27].) distribution of the $\tau$ decays, and hence contains information on $\left|V_{u s}\right|$. The hadronic vacuum polarization function $\Delta \Pi_{\tau}$ is evaluated by the operator product expansion (OPE). (Here, " $\Delta$ " indicates the difference between $\tau \rightarrow X_{s} v_{\tau}$ and $X_{d} v_{\tau}$ decays.) In the conventional set up [24], the weight function is set to the kinematical factor $w(s)=(1-y)^{2}(1+2 y)\left(y=s / m_{\tau}^{2}\right), s_{0}=m_{\tau}^{2}$, and the vaccum 
saturation approximation (VSA) is assumed to evaluate the higher order corrections in the OPE. However, Eq. (2.8) should be valid for any $s_{0}$ and analytic $w(s)$.

K. Maltman presented a detailed study of theoretical uncertainties [26]. As shown in Fig. 4, $\left|V_{u s}\right|$ estimated in the conventional setup significantly depends on the choice of $w(s)$ and $s_{0}$. This dependence is largely reduced in a new implementation, in which both $\left|V_{u s}\right|$ and non-perturbative parameters at higher orders in the OPE are fixed from experimental inputs [27]. Together with preliminary $\mathrm{BaBar}$ estimate of $\mathrm{BR}\left(\tau \rightarrow K^{-} \pi^{0} v_{\tau}\right)$ [28], the new implementation yields $\left|V_{u s}\right|=$ $0.2228(23)_{\mathrm{ex}}(5)_{\text {th }}$ [26], which is in good agreement with Eq. (2.7).

Future improvements of the experimental inputs are important to be competitive with the determination from the kaon decays. The current experimental status is summarized by S. Banerjee [29]. Another strategy is proposed in Refs. [26, 30,31] to use designed weights and lattice QCD data of the vacuum polarization function.

\subsection{CKM unitarity in the first row}

Reference [17] combines $\left|V_{u d}\right|$ from the superallowed nuclear decays, $\left|V_{u s}\right|$ and $\left|V_{u s}\right| /\left|V_{u d}\right|$ from the $K_{\ell 3}$ and $P_{\ell 2}$ decays in a single fit. With the hadronic inputs from $N_{f}=2+1+1$ lattice QCD, this fit yields $\left|V_{u d}\right|=0.97418(21)$ and $\left|V_{u s}\right|=0.2246(5)$, which are consistent with Eqs. (2.2) and (2.7). A measure of the CKM unitarity violation is estimated as

$$
\begin{aligned}
\Delta_{\mathrm{CKM}} & =\left|V_{u d}\right|^{2}+\left|V_{u s}\right|^{2}+\left|V_{u b}\right|^{2}-1 \\
& =-0.0005(5),
\end{aligned}
$$

where $\left|V_{u b}\right| \approx 4 \times 10^{-3}$ has negligibly small contribution. This does not change significantly $\left(\Delta_{\mathrm{CKM}}=\right.$ $-0.0006(5))$ when we employ the lattice input for $N_{f}=2+1$. The current determination of $\left|V_{u d}\right|$ and $\left|V_{u s}\right|$, therefore, confirms the unitarity in the first row at $0.1 \%$ accuracy. Model independent analyses based on effective field theory suggest that this precision test is sensitive to new physics with typical scale of $\lesssim 10 \mathrm{TeV}[32,33]$.

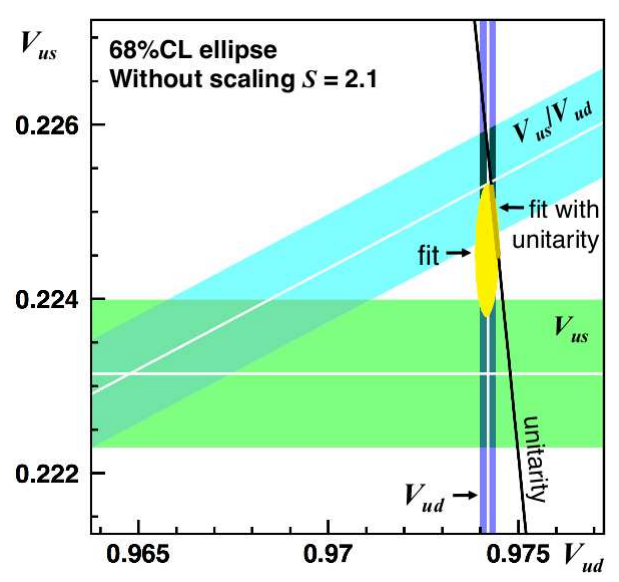

Figure 5: Test of CKM unitarity in the first row. The yellow region is obtained from a single fit to $\left|V_{u d}\right|,\left|V_{u s}\right|,\left|V_{u s}\right| /\left|V_{u d}\right|$ from the nuclear, $K_{\ell 3}$ and $P_{\ell 2}$ decays (inputs from $N_{f}=$ $2+1+1$ lattice QCD are used for the latter two). This region is consistent with the black solid line, which satisfies CKM unitarity. (Figure from Ref. [17].)

\section{3. $\left|V_{c d}\right|,\left|V_{c s}\right|$ and charm (semi)leptonic decays}

\subsection{Leptonic decays}

Large data samples of charm decays have been collected by CLEO-c and BESIII at the $\psi(3770)$ and by the $B$ factories, Belle and BaBar, at the $\Upsilon(4 S)$. Branching fractions of leptonic decays of $D_{q}$ mesons are expressed as

$$
\operatorname{BR}\left(D_{q}^{+} \rightarrow \ell^{+} v\right)=\frac{G_{F}^{2}}{8 \pi} \tau_{D_{q}} f_{D_{q}}^{2}\left|V_{c q}\right|^{2} M_{D_{q}} m_{\ell}^{2}\left(1-\frac{m_{\ell}^{2}}{M_{D_{q}}^{2}}\right)^{2},
$$



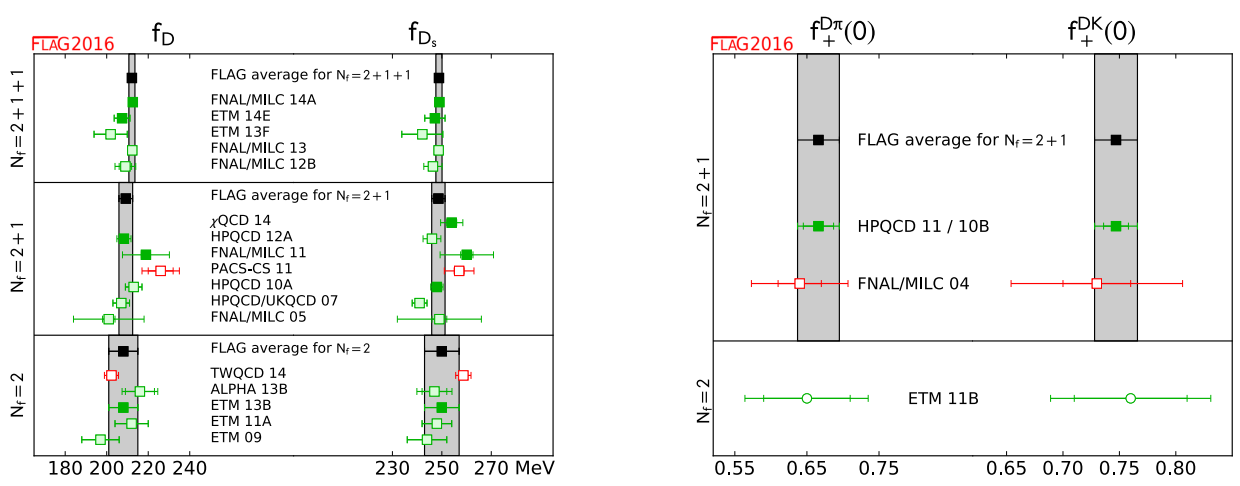

Figure 6: Lattice QCD calculations of decay constants $f_{D_{(s)}}$ (left panel) and $D \rightarrow K(\pi)$ semileptonic form factors $f_{+}(0)^{D K(\pi)}$ at $q^{2}=0$ (right panel). (Figure from Ref. [16].)

where $\tau_{D_{q}}$ and $f_{D_{q}}$ represent the lifetime and decay constant of $D_{q}$ meson, respectively.

As discussed by J.T. Tsang [34], the lattice QCD determination of $f_{D_{q}}$ has been largely improved by realistic simulations near the physical quark masses on fine lattices. In particular, the latter enables us to use relativistic charm quark actions with good control of discretization errors and renormalization of $f_{D_{q}}$. As shown in the left panel of Fig. 6, there have been independent calculations with different lattice actions. The accuracy of the world average is $0.7(0.5) \%$ for $f_{D}\left(f_{D_{s}}\right)$. As in the case of the kaon decays, the isospin and EM corrections start to be relevant at this level of accuracy. Reference [35] presented a lattice QCD estimate of the isospin correction $f_{D^{+}}-f_{D^{0}}=0.47\left(\begin{array}{c}+25 \\ -6\end{array}\right) \mathrm{MeV}$. We also note that the method of Ref. [20] can be also applied to the $D_{q}$ meson decays.

H. Ma reviewed recent experimental progress [36]. A measurement of the $\operatorname{BR}\left(D^{+} \rightarrow \mu^{+} v\right)$ has been performed by BESIII using $2.93 \mathrm{fb}^{-1} \psi(3770)$ data set, from the recoil of tagged $D^{-}$mesons [37]. The result has 5\% accuracy, which is dominated by the statistical uncertainty from about 400 signal events. The accuracy of this measurement will be improved significantly if $10 \mathrm{fb}^{-1}$ additional data is taken by BESIII. Preliminary result of the first measurement of $\operatorname{BR}\left(D^{+} \rightarrow \tau^{+} v\right)$ has also been presented by BESIII, which gives a ratio $\operatorname{BR}\left(D^{+} \rightarrow \tau^{+} v\right) / \operatorname{BR}\left(D^{+} \rightarrow \mu^{+} v\right)=3.21 \pm 0.64$, compatible with the SM prediction. Results for $\operatorname{BR}\left(D_{s} \rightarrow \ell^{+} v\right)$ have been obtained in the $\mu$ and $\tau$ channels by BaBar and Belle using the full data sample [38, 39], and by BESIII using $482 \mathrm{pb}^{-1}$ data at center-of-mass energy of $4.009 \mathrm{GeV}$ [40]. The world average values are $\mathrm{BR}\left(D_{s} \rightarrow \mu^{+} v\right)=$ $(5.54 \pm 0.23) \times 10^{-3}$ and $\operatorname{BR}\left(D_{s} \rightarrow \tau^{+} v\right)=(5.51 \pm 0.24) \times 10^{-2}$ [41]. Improved measurements are expected from Belle II and BESIII using more data in the near future.

\subsection{Semileptonic decays}

The $D \rightarrow P \ell v$ semileptonic decays, where $P$ represents the final state pseudoscalar meson, proceed through the weak vector current. The relevant matrix element is parameterized as in Eq. (2.5). The contribution from the scalar form factor $f_{0}^{D P}$ is suppressed by the lepton mass squared $m_{\ell}^{2}$, and can be neglected for light leptons. The differential decay rate is given by

$$
\frac{d \Gamma(D \rightarrow P \ell v)}{d q^{2}}=\frac{G_{F}^{2}}{24 \pi^{3}} p_{P}^{3}\left|f_{+}^{D P}\left(q^{2}\right)\right|^{2}\left|V_{c q}\right|^{2},
$$



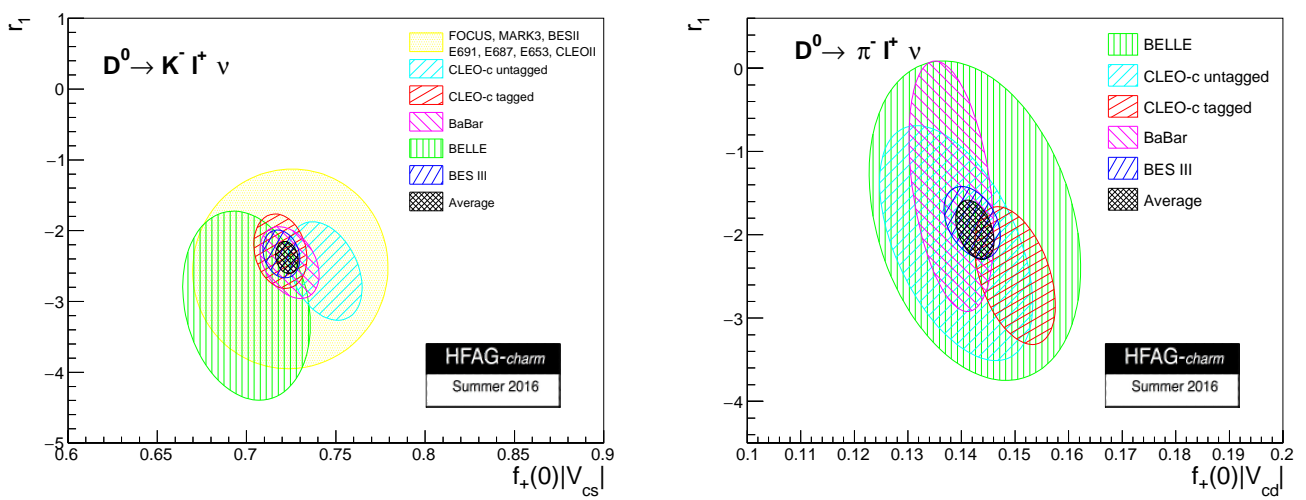

Figure 7: Experimental results for $f_{+}^{D P}(0)\left|V_{c q}\right|$ (horizontal axis) and a ratio of coefficients in $z$-parameter expansion (vertical axis). The left and right panels are for the $D \rightarrow K \ell v$ and $D \rightarrow \pi \ell v$ channels, respectively. Results obtained by different experiments and the HFAG averages are plotted. (Figure from Ref. [41].)

where $p_{P}$ is the momentum of $P$ in the $D$ rest frame. The $q^{2}$ dependence of $f_{+}^{D P}$ has been often parameterized as a sum of effective poles, including the lowest-lying $c \bar{q}$ resonance with appropriate quantum numbers. It is popular in recent analyses to use the so-called $z$-parameter expansion [42], which is a model independent parameterization based on the analyticity of $f_{+}^{D P}$. Experiments determine the parameters describing the form factor shape (effective pole masses, coefficients of $z$-parameter expansion) and the normalization of the form factor at $q^{2}=0$ times the CKM matrix element, namely $f_{+}^{D P}(0)\left|V_{c q}\right|$.

Recent experimental progress is reviewed by A. Soffer [43] and Y. Zheng [44]. Figure 7 compares the results from different experiments [41]. The BaBar experiment has recently analysed the $D^{0} \rightarrow \pi^{-} e^{+} v$ channel [45]. The most precise values for the $D^{0} \rightarrow K^{-} \ell^{+} v$ and $D^{0} \rightarrow \pi^{-} \ell^{+} v$ channels are obtained by the BESIII experiment using $2.93 \mathrm{fb}^{-1} \psi(3770)$ data set [46]. Combined results of measured form factors by several experiments have reached an accuracy of $0.5 \%$ and $1 \%$ for the Cabibbo-allowed and suppressed modes, respectively [41]. Results for the $D^{+}$channel, with $K_{S}, K_{L}$ and $\pi^{0}$ in the final state, have also been reported by BESIII, although they are less precise than the ones obtained for $D^{0}$ channel. Results support isospin conservation. Other interesting modes with a vector in the final state, $D \rightarrow\left\{K^{*}, \omega, \phi\right\} \ell v$, and $D_{s} \rightarrow\left\{\phi, \eta, \eta^{\prime}\right\} \ell v$ are also being measured [44]. Furthermore, BESIII performed the first absolute measurement of $\operatorname{BR}\left(\Lambda_{c}^{+} \rightarrow \Lambda \ell^{+} v\right)$ based on $0.567 \mathrm{fb}^{-1}$ data at $4.6 \mathrm{GeV}$ (near the $\Lambda_{c}^{+} \bar{\Lambda}_{c}^{-}$mass threshold), which has precision of $12 \%$. We expect 10 times more $\Lambda_{c}^{+}$data in the coming years at BESIII and the precision reduced to being less than $4 \%$.

As discussed by A. Davis [47], the LHCb experiment could also contribute in the near future in measuring charm semileptonic branching fractions and CKM matrix elements. With the $3 \mathrm{fb}^{-1}$ collected in $p p$ collisions during Run-I, about $5 \times 10^{6}$ of signal events are expected for the Cabibboallowed mode. Neutrino reconstruction in this environment is challenging, but information on the $D$ flight direction and track momenta can be used to constraint the neutrino transverse momentum in a similar manner as in $b$-hadron semileptonic decays [48].

In contrast to the calculation for the kaon decays (Fig. 3), there have been only few lattice esti- 
mates of the the form factor normalization $f_{+}^{D P}(0)$ as shown in the right panel of Fig. 6. The accuracy is roughly $3 \%[49,50]$, which is much larger than the experimental accuracy for $f_{+}^{D P}(0)\left|V_{c q}\right|$. However, the lattice determination can be straightforwardly improved by more realistic simulations with relativistic charm quark actions. Indeed, as reviewed by E. Gámiz [51], there are independent on-going lattice calculations [52, 53, 54]. In addition, lattice QCD data are available over the whole $q^{2}$ region [55], and become more precise towards the zero recoil limit. Therefore, the accuracy of $\left|V_{c q}\right|$ from the semileptonic decays is expected to be significantly improved by a global fit of all experimental and lattice data using a model independent parametrization, such as the $z$-parameter expansion. We also note that a first lattice calculation of the $\Lambda_{c} \rightarrow \Lambda$ semileptonic form factors became available [56]. These baryonic decays may offer an independent determination of $\left|V_{c s}\right|$ in the future.

\section{3 $\left|V_{c d}\right|,\left|V_{c s}\right|$ and search for new physics}

With the above-mentoned progress, the CKM matrix elements

$$
\left|V_{c s}\right|=0.997(17), \quad\left|V_{c d}\right|=0.216(5)
$$

are extracted from the charm leptonic and semileptonic decays [41]. This confirms the unitarity in the second row with an accuracy of $7 \%(2 \sigma)$

$$
\left|V_{c d}\right|^{2}+\left|V_{c s}\right|^{2}+\left|V_{c b}\right|^{2}=1.042(34),
$$

where $\left|V_{c b}\right| \approx 4 \times 10^{-2}$ has only small effect.

As discussed by S. Fajfer [57], present accuracy of the theoretical and experimental inputs offers the possibility to search for new physics in $c \rightarrow s$ transitions, by looking for small deviations from the SM predictions in several observables such as branching ratios, forward-backward asymmetry in $D \rightarrow K \ell v$ decays and transversal muon polarization [58, 59]. In addition, tests of lepton flavor universality in charm decays are also proposed $[59,60]$ that use the $\operatorname{BR}\left(D^{+} \rightarrow\right.$ $\left.\pi^{+} \mu^{+} \mu^{-}\right) / \operatorname{BR}\left(D^{+} \rightarrow \pi^{+} e^{+} e^{-}\right)$ratio in different $q^{2}$ bins. The SM prediction for this observable has a per mille accuracy in the range of $[1.25-1.73] \mathrm{GeV}^{2}$. Predicted limits for lepton flavor violation $c \rightarrow u \ell \ell^{\prime}$ are also available [60].

\section{Conclusions}

As reported in the WG1 sessions, there have been important theoretical and experimental progress in the determination of $\left|V_{u d}\right|,\left|V_{u s}\right|,\left|V_{c d}\right|$ and $\left|V_{c s}\right|$. CKM unitarity is now confirmed with $0.1 \%$ and $7 \%$ precisions in the first and second rows, respectively.

The accuracy of $\left|V_{u d}\right|$ and $\left|V_{u s}\right|$ is rather stable in recent years. However, reliability has been steadily improved by, for instance, a thorough test of the isospin corrections to the super-allowed nuclear decays, and by resolving the long-standing puzzle on $\left|V_{u s}\right|$ from the inclusive hadronic $\tau$ decays. A long-standing challenge towards a more stringent unitarity test is improving the calculation of the transition-independent radiative correction to the nuclear decays. Recent remarkable progress in precision lattice calculations of kaon matrix elements may justify renewed experimental efforts on the kaon (semi)leptonic decays [61]. 
Experiments and lattice QCD are in a healthy competition towards high precision study of charm decays leading to recent rapid improvemenet in the determination of $\left|V_{c d}\right|$ and $\left|V_{c s}\right|$. There are good prospects for future experimental progress by BESIII, Belle II and possibly LHCb. In the near future, we expect significant improvement in the lattice determination of the semileptonic charm decay form factors. The accuracy of other hadronic inputs for $K$ and $D$ decays are now below $1 \%$. At this level, isospin and EM corrections have to be taken into account in a controlled way. The lattice QCD determination of these correction is under active development.

The work of TK is supported in part by the Grant-in-Aid of the MEXT (No. 26400259) and by MEXT as "Priority Issue on Post-K computer" (Elucidation of the Fundamental Laws and Evolution of the Universe) and JICFuS.

\section{References}

[1] R.E. Behrends and A. Sirlin, Phys. Rev. Lett. 4 (1960) 186.

[2] M. Ademollo and R. Gatto, Phys. Rev. Lett. 13 (1964) 264.

[3] J.C. Hardy and I.S. Towner, PoS (CKM2016) 028.

[4] A.A. Valverde et al., Phys. Rev. Lett. 114 (2015) 232502 [arXiv:1503.08124 [nucl-ex]].

[5] P.A. Voytas, E.A. George, G.W. Severin, L. Zhan and L.D. Knutson, Phys. Rev. C92 (2015) 065502 [arXiv:1512.00430 [nucl-ex]].

[6] M.R. Dunlop et al., Phys. Rev. Lett. 116 (2016) 172501.

[7] I.S. Towner and J.C. Hardy, Phys. Rev. C82 (2010) 065501 [arXiv:1007.5343 [nucl-th]].

[8] D. Počanić, PoS (CKM2016) 029 [arXiv:1704.00192 [nucl-ex]].

[9] F.E. Wietfeldt and G.L. Greene, Rev. Mod. Phys. 83 (2011) 1173.

[10] S. Baeßler, J.D. Bowman, S. Penttilä and D. Počanić, J. Phys. G 41 (2014) 114003 [arXiv:1408.4737 [nucl-ex]].

[11] A. Young et al., J. Phys. G 41 (2014) 114007.

[12] http://nab.phys.virginia.edu (Nab experiment).

[13] D.H. Wilkinson, Nucl. Phys. A377 (1982) 474.

[14] W.J. Marciano, Phys. Rev. Lett. 93 (2004) 231803 [arXiv:hep-ph/0402299].

[15] M. Antonelli et al. (FlaviaNet Working Group on Kaon Decays), Eur. Phys. J. C69 (2010) 399 [arXiv:1005.2323 [hep-ph]].

[16] S. Aoki et al. (Flavor Lattice Averaging Group), Eur. Phys. J. C77 (2017) 112 [arXiv:1607.00299 [hep-lat]].

[17] M. Moulson, PoS (CKM2016) 033 [arXiv:1704.04104 [hep-ex]].

[18] S. Simula, PoS (CKM2016) 032 [arXiv:1704.00510 [hep-lat]].

[19] S. Aoki et al. (Flavor Lattice Averaging Group), http://itpwiki.unibe.ch/flag (the web update of Ref. [16]).

[20] N. Carrasco et al., Phys. Rev. D91 (2015) 074506 [arXiv:1502.00257 [hep-lat]]. 
[21] V. Lubicz et al., PoS (LATTICE 2016) 290 [arXiv:1610.09668 [hep-lat]].

[22] V. Cirigliano and H. Neufeld, Phys. Lett. B700 (2011) 7 [arXiv:1102.0563 [hep-ph]].

[23] J.L. Rosner, S. Stone, R.S. Van de Water, arXiv:1509.02220 [hep-ph] (prepared for the 2016 Particle Data Book).

[24] E. Gámiz, M. Jamin, A. Pich, J. Prades and F. Schwab, JHEP 0301 (2003) 060 [arXiv:hep-ph/0212230 [hep-ph]].

[25] Y. Amhis et al. (Heavy Flavor Averaging Group), arXiv:1412.7515 [hep-ex].

[26] K. Maltman et al., PoS (CKM2016) 030.

[27] K. Maltman, R.J. Hudspith, R. Lewis, C.E. Wolfe and J. Zanotti, J. Univ. Sci. Tech. China 46 (2016) 374 [arXiv:1511.08514 [hep-ph]].

[28] A. Adametz, Universtity of Heidelberg PhD thesis, July 2011.

[29] S. Banerjee, ' $\left|V_{u s}\right|$ from $\tau$ decays', presented at the 9th International Workshop on the CKM Unitarity Triangle (CKM2016) http: / /www.tifr.res.in/ ckm16/.

[30] K. Maltman et al., PoS (LATTICE2016) 279.

[31] H. Ohki et al. PoS (LATTICE2016) 288.

[32] V. Cirigliano, J.P. Jenkins and M. González-Alonso, Nucl. Phys. B830 (2010) 95 [arXiv:0908.1754 [hep-ph]].

[33] M. González-Alonso, J.M. Camalich, JHEP 1612 (2016) 052 [arXiv:1605.07114 [hep-ph]].

[34] J.T. Tsang, PoS (CKM2016) 022 [arXiv:1702.05360 [hep-lat]].

[35] A. Bazavov et al. (FNAL/MILC Collaboration), Phys. Rev. D90 (2014) 074509 [arXiv: 1407.3772 [hep-lat]].

[36] H. Ma, PoS (CKM2016) 021.

[37] M. Ablikim et al. (BESIII Collaboration), Phys. Rev. D89 (2014) 051104 [arXiv:1312.0374 [hep-ex]].

[38] P. del Amo Sanchez et al. (BaBar Collaboration), Phys. Rev. D82 (2010) 091103 Erratum: [Phys. Rev. D 91 (2015), 019901] [arXiv:1008.4080 [hep-ex]].

[39] A. Zupanc et al. (Belle Collaboration), JHEP 1309 (2013) 139 [arXiv:1307.6240 [hep-ex]].

[40] M. Ablikim et al. (BESIII Collaboration), Phys. Rev. D94 (2016), 072004 [arXiv:1608.06732 [hep-ex]].

[41] Y. Amhis et al., HFAG 2016, arXiv:1612.07233 [hep-ex].

[42] C. Bourrely, B. Machet and E. de Rafael, Nucl. Phys. B189 (1981) 157.

[43] A. Soffer, ' $D \rightarrow P e^{+} v_{e}$ form factors, $\left|V_{c s}\right|$ and $\left|V_{c d}\right|$ ', presented at the 9th International Workshop on the CKM Unitarity Triangle (CKM2016) http://www.tifr.res.in/ Ckm16/.

[44] Y. Zheng, 'Experimental measurements of $D_{(s)}$ semileptonic branching fractions', presented at the 9th International Workshop on the CKM Unitarity Triangle (CKM2016) http://www.tifr.res.in/ ckm16/.

[45] J. P. Lees et al. (BaBar Collaboration), Phys. Rev. D91 (2015) 052022 [arXiv:1412.5502 [hep-ex]].

[46] M. Ablikim et al. (BESIII Collaboration), Phys. Rev. D92 (2015) 072012 [arXiv:1508.07560 [hep-ex]]. 
[47] A. Davis, PoS (CKM2016) 025.

[48] R. Aaij et al. [LHCb Collaboration], Nature Phys. 11 (2015) 743 [arXiv:1504.01568 [hep-ex]].

[49] H. Na, C.T.H. Davies, E. Follana, G.P. Lepage and J. Shigemitsu (HPQCD Collaboration), Phys. Rev. D82 (2010) 114506 [arXiv:1008.4562 [hep-lat]].

[50] H. Na et al. (HPQCD Collaboration), Phys. Rev. D84 (2011) 114505 [arXiv:1109.1501 [hep-lat]].

[51] E. Gámiz, ' $D$-meson semileptonic decays with lattice QCD', presented at the 9th International Workshop on the CKM Unitarity Triangle (CKM2016) http://www.tifr.res.in/ ckm16/.

[52] V. Lubicz, L. Riggio, G. Salerno, S. Simula and C. Tarantino (ETM Collaboration), PoS (LATTICE2016) 280 [arXiv:1611.00022 [hep-lat]].

[53] T. Kaneko, B. Fahy, H. Fukaya and S. Hashimoto (JLQCD Collaboration), PoS (LATTICE2016) 297 [arXiv:1701.00942 [hep-lat]].

[54] T. Primer et al. (FNAL/MILC Collaboration), PoS (LATTICE2016) 305.

[55] J. Koponen et al. (HPQCD Collaboration), arXiv:1305.1462 [hep-lat].

[56] S. Meinel, Phys. Rev. Lett. 118 (2017) 082001 [arXiv:1611.09696 [hep-lat]].

[57] S. Fajfer, 'New physics search in $D$ meson decays', presented at the 9th International Workshop on the CKM Unitarity Triangle (CKM2016) http://www.tifr.res.in/ ckm16/.

[58] J. Barranco, D. Delepine, V. Gonzalez Macias and L. Lopez-Lozano, arXiv:1404.0454 [hep-ph].

[59] S. Fajfer, I. Nisandzic and U. Rojec, Phys. Rev. D91 (2015) 094009 [arXiv:1502.07488 [hep-ph]].

[60] S. de Boer and G. Hiller, Phys. Rev. D93 (2016) 074001 [arXiv:1510.00311 [hep-ph]].

[61] A. Ceccucci, 'Kaon experiments', presented at the 9th International Workshop on the CKM Unitarity Triangle (CKM2016) http: / /www.tifr.res.in/ ckm16/. 